\title{
Avaliação de ansiedade e depressão em pacientes oncológicos: comparação psicométrica
}

\author{
Cristiane Decat Bergerot - Universidade de Brasilia, Brasilia, Brasil \\ Jacob Arie Laros - Universidade de Brasilia, Brasilia, Brasil \\ Tereza Cristina Cavalcanti Ferreira de Araujo - Universidade de Brasilia, Brasília, Brasil
}

\begin{abstract}
Resumo
Instrumentos de avaliação de ansiedade e depressão são úteis para diagnóstico e orientação do manejo clínico diante das alterações emocionais suscitadas pelas vivências do câncer. O presente estudo comparou vantagens e desvantagens psicométricas de instrumentos comumente utilizados em serviços especializados em Oncologia: Escala de Ansiedade e Depressão (HADS), Transtorno Geral de Ansiedade (GAD-7) e Questionário sobre Saúde do Paciente (PHQ-9). Participaram da pesquisa, 200 pacientes diagnosticados com câncer, em tratamento quimioterápico, sendo 30,5\% homens e 69,5\% mulheres, com idade entre 18 a $89 \operatorname{anos}(M=56,8 ; D P=15)$. Os instrumentos mostraram coeficientes de fidedignidade variando entre 0,74 e 0,84 . As características psicométricas estudadas indicaram valores melhores para HADS-D e GAD-7. Entretanto, HADS-A e PHQ-9 também se mostraram adequados para avaliação de ansiedade e depressão. Sugere-se a adoção desses instrumentos para triagem, diagnóstico e monitoramento de pacientes com câncer, especialmente nos domínios psicológico e social.

Palavras-chave: Câncer; Ansiedade; Depressão; Psicometria; Medidas estatísticas.
\end{abstract}

Assessment of anxiety and depression in cancer patients: A psychometric comparison

\begin{abstract}
Evaluation tools of anxiety and depression are useful for diagnosis and clinical management orientation before the changes caused by cancer experiences. The present study compared psychometric advantages and disadvantages of instruments commonly used in specialized services in Oncology: Hospital Anxiety and Depression Scale (HADS), General Anxiety Disorder (GAD-7) and the Patient Health Questionnaire (PHQ-9). There were 200 patients diagnosed with cancer and in chemotherapy treatment participating in the study, being 30.5\% men and 69.5\% women, aged between 18 to 89 years $(M=56.8$; $S D=15)$. The instruments showed coefficients of reliability varying between .74 and .84 . The evaluated psychometric characteristics indicated better values for HADS-D and GAD-7. However, HADS-A and PHQ-9 were also found to be suitable for evaluation of anxiety and depression. We suggest the adoption of these instruments for screening, diagnosis and monitoring of cancer patients, especially in psychological and social domains.

Keywords: Cancer; Anxiety; Depression; Psychometrics; Statistical measurement.
\end{abstract}

\section{Evaluación de la ansiedad y la depresión en pacientes oncológicos: comparación psicométrica}

\begin{abstract}
Resumen
Instrumentos de evaluación de ansiedad y depresión son útiles para el diagnóstico y la orientación del manejo clínico frente a los cambios emocionales originados por las experiencias del cáncer. Este estudio comparó ventajas y desventajas psicométricas de instrumentos utilizados comúnmente en servicios especializados en Oncología: Escala de Ansiedad y Depresión (HADS), Trastorno General de Ansiedad (GAD-7) y Cuestionario sobre la Salud del Paciente (PHQ-9). Participaron del estudio 200 pacientes diagnosticados con cáncer, en tratamiento con quimioterapia, siendo 30,5\% hombres y $69,5 \%$ mujeres, con edades entre 18 y 89 años $(M=56,8 ; D T=15)$. Los instrumentos mostraron índices de confiabilidad variando entre 0,74 y 0,84 . Las características psicométricas estudiadas mostraron mejores valores para la HADS-D y el GAD-7. Sin embargo, la HADS-A y el PHQ-9 también se mostraron adecuados para la evaluación de la ansiedad y la depresión. Se sugiere adoptar estos instrumentos para la detección, el diagnóstico y el seguimiento de pacientes con cáncer, especialmente en los dominios psicológicos y sociales. Palabras-clave: Cáncer; Ansiedad; Depresión; Psicometría; Medidas estadísticas.
\end{abstract}

Em oncologia, o acompanhamento clínico revela sintomas de ansiedade e depressão desde a fase diagnóstica do(s) câncer(es). Observam-se, também, repercussões dessas reações psicológicas na adesão ao tratamento, duração das internações, qualidade de vida, prognóstico e sobrevida à doença (Bultz \& Johansen, 2011; Dalton e cols., 2009; Stark e cols., 2002). Aproximadamente 20 a $48 \%$ dos pacientes oncológicos apresentam critérios diagnósticos para ansiedade e/ ou para depressão (Ballenger e cols., 2001; Levin \&
Alici, 2010; Miller \& Massie, 2010; Stark e cols., 2002). Soma-se a isso, a constatação de que, frequentemente, depressão coexiste com quadros de ansiedade (Massie, 1989). Um estudo realizado pela National Comorbidity Survey mostrou que $51 \%$ dos pacientes diagnosticados com depressão e ansiedade manifestaram exacerbação de sintomas, recuperação prolongada e mais readmissões em serviço de saúde (Grady-Weliky, 2002; Massie, 2004). Diante de tais evidências, instituições internacionais recomendam a utilização de protocolos para 
triagem, diagnóstico e monitoramento de parâmetros psicológicos e sociais (Adler \& Page, 2008; Jacobsen e cols., 2012).

No que tange à ansiedade e depressão, uma variedade de instrumentos têm sido indicados para avaliação em oncologia, como por exemplo: Beck Anxiety Inventory (BAI); Beck Depression Inventory (BDI-II e BDI-SF); Brief Symptom Inventory (BSI); Generalized Anxiety Disorder Screener (GAD-7); General Health Questionnaire (GHQ); Hospital Anxiety and Depression Scale (HADS); Impact of Events Scale (IES); Patient Health Questionnaire (PHQ-9); e Profile of Mood States (POMS) (Miller \& Massie, 2010; Mitchell, 2010; Noyes Jr., Holt \& Massie, 1998).

A despeito do uso frequente da Escala de Ansiedade e Depressão (HADS), Transtorno Geral de Ansiedade (GAD-7) e Questionário sobre a Saúde do Paciente (PHQ-9) - instrumentos de aplicação breve e já validados - não existe consenso quanto ao seu interesse clínico em oncologia, uma vez que os resultados atinentes às suas características psicométricas são contraditórios (Mitchell, 2010; Noyes Jr. e cols., 1998). Cumpre destacar que, em carta publicada na revista Journal of Psychosomatic Research, contestou-se o emprego da HADS para avaliação de ansiedade e depressão. Os autores apoiaram seu julgamento em uma revisão sistemática, divulgada no mesmo periódico, na qual se comprovou forte heterogeneidade dos resultados e falha na estrutura dos itens (Cosco e cols., 2012; Coyne \& Sonderen; 2012a, 2012b).

Ainda quanto à HADS, Razavi, Delvaux, Farvacques e Robaye (1990) verificaram, por meio da análise fatorial, que apenas um fator é avaliado por esse instrumento, tratando-se, portanto, de uma escala unidimensional. $\mathrm{Na}$ análise de Moorey e cols. (1991) identificaram-se dois fatores e, nos estudos de Brandberg e cols. (1992) e de Rodgers e cols. (2005), três fatores foram encontrados. Outros trabalhos identificaram melhor performance para a subescala de ansiedade (HADS-A), quando comparada à de depressão (HADS-D) (Hall e cols., 1999; Mystakidou e cols., 2007). Tais evidências suscitam a necessidade de mais pesquisas, inclusive com amostras brasileiras (Baker-Glenn, Mitchell \& Symonds, 2008; Lambert e cols., 2013; Pirl, 2010).

Tanto a GAD-7 quanto o PHQ-9 são ferramentas amplamente adotadas para triagem diagnóstica de transtornos mentais (Spitzer, Kroenke \& Williams, 1999). A literatura identificou sensibilidade de $89 \%$ (GAD-7) e 82\% (PHQ-9), e especificidade de $82 \%$ (GAD-7) e 88\% (PHQ-9) para o escore 10 (Kroenke, Spitzer \& Williams, 2001; Spitzer, Kroenke, Williams \&
Löwe, 2006), resultado confirmado em recente revisão publicada (Kroenke e cols., 2010). Em suma, tendo em vista a relevância assistencial e científica da temática, realizou-se uma investigação com o objetivo geral de avaliar vantagens e desvantagens psicométricas e clínicas de medidas usualmente empregadas em serviços de oncologia (HADS, GAD-7 e PHQ-9).

\section{Método}

\section{Participantes}

Constituiu-se uma amostra de conveniência com 200 pacientes adultos que, de acordo com os critérios de inclusão: (1) realizaram tratamento integral no centro especializado em que a investigação foi empreendida; e (2) manifestaram concordância em participar por meio de assinatura no Termo de Consentimento Livre e Esclarecido. Vale comentar que não houve recusa em participar do estudo. Estipularam-se como critérios de exclusão: (1) idade inferior a 18 anos; (2) iniciar tratamento quimioterápico em outro serviço; e (3) pacientes estrangeiros sem domínio do idioma português.

\section{Instrumentos}

(1) Questionário Sociodemográfico - abrange os tópicos idade, sexo, estado civil, nível de escolaridade, diagnóstico e estadiamento da doença.

(2) HADS - escala traduzida e validada por Botega, Bio, Zomignani, Garcia Jr. e Pereira (1995), visa detectar graus leves de transtornos afetivos em ambientes não psiquiátricos. É constituída por 14 itens de múltipla escolha, dos quais sete são voltados para avaliação da ansiedade (HADS-A) e sete para depressão (HADS-D). Cada item pode ser pontuado de 0 a 3 , atingindo-se máximo de 21 pontos em cada subescala. $\mathrm{Na}$ presente pesquisa, adotaram-se os pontos de corte informados na literatura: escore indicativo de ansiedade igual ou maior que 8; e escore indicativo de depressão igual ou superior a 9 .

(3) GAD-7 - instrumento breve para avaliação, diagnóstico e monitoramento de ansiedade, foi elaborado por Spitzer e cols. (2006) e validado por Kroenke, Spitzer, Williams, Monahan e Löwe (2007), de acordo com os critérios do Manual Diagnóstico e Estatístico de Transtornos Mentais (DSM-IV). A tradução para a língua portuguesa foi feita por Pfizer (Copyright (C) 2005 Pfizer Inc., New York, NY), com registro de evidência de validade no Brasil (Mapi Research Institute, 2006). É composta por sete itens, dispostos em uma escala de quatro pontos: 0 (nenhuma vez) a 3 (quase todos os 
dias), com pontuação que varia de 0 a 21, ao medir frequência de sinais e sintomas de ansiedade nas últimas duas semanas. Considera-se indicador positivo de sinais e sintomas de transtornos de ansiedade, valor igual ou maior que 10 .

(4) PHQ-9 - instrumento breve para avaliação, diagnóstico e monitoramento de transtorno depressivo, de acordo com os critérios do DSM-IV. A evidência de validade foi verificada por Spitzer e cols. (1999) e por Kroenke e cols. (2001) e a tradução para a língua portuguesa foi veiculada pela Pfizer (Copyright (C) 2005 Pfizer Inc., New York, NY). No Brasil, Osório, Mendes, Crippa e Loureiro (2009) constataram a evidência de validação. $\mathrm{O}$ instrumento reúne nove itens, dispostos em uma escala de quatro pontos: 0 (nenhuma vez) a 3 (quase todos os dias), com pontuação que varia de 0 a 27 para avaliar a frequência de sinais e sintomas de depressão nas últimas duas semanas. Estima-se, como indicador positivo de depressão maior, valor maior ou igual a 10 .

\section{Procedimentos de coleta e análise dos dados}

A coleta de dados foi realizada como parte da rotina de atendimento do setor de Psico-Oncologia no referido centro especializado. Destarte, no primeiro dia do ciclo de tratamento quimioterápico, a pesquisadora e psicóloga responsável aplicou os instrumentos selecionados, na seguinte ordem: Questionário Sociodemográfico, HADS, GAD-7 e PHQ-9.

Utilizou-se análise descritiva para caracterização dos dados sociodemográficos e clínicos da amostra, bem como para distribuição dos participantes de acordo com os escores de ansiedade e depressão. A avaliação da consistência interna dos instrumentos foi feita a partir do cálculo do coeficiente de fidedignidade (lambda2) e da correlação média entre os itens (Tabachnick \& Fidell, 2007). No cálculo do índice de fidedignidade, foi utilizado o coeficiente lambda2 de Guttman, tendo em vista que este índice estima melhor a fidedignidade do que o coeficiente alfa de Cronbach, quando os instrumentos contêm poucos itens ou quando a amostra é pequena, sendo calculado o erro padrão da média (Tellegen \& Laros, 2004; Ten Berge \& Zegers, 1978). Realizou-se também análise fatorial exploratória das escalas separadamente - utilizando o Principal Axis Factoring (PAF) e uma análise fatorial das escalas em conjunto -, para obter evidências de validade discriminante das escalas (Cattell, 1966; Costello \& Osborne, 2005; Damásio, 2012; Laros, 2012; Smith \& McCarthy, 1995). Este método é adequado, segundo Clark e Watson (1995), para extrair evidências de validade discriminante das escalas. Nessa análise, foi utilizada novamente a PAF com promax. Por último, verificou-se a correlação existente entre as escalas, com intervalo de confiança de 95\%. Todas essas análises foram feitas com o auxílio do software Statistical Package for the Social Sciences (SPSS 17.0).

\section{Resultados}

A Tabela 1 apresenta a caracterização sociodemográfica da amostra estudada. Verificou-se que 30,5\% dos pacientes eram do sexo masculino, com idade entre 18 e 89 anos $(M=56,8$ anos; $D P=15)$, sendo $63 \%$ casados. O grau de escolaridade variou entre ensino fundamental $(14,5 \%)$ e pós-graduação $(9 \%)$. Quanto às categorias diagnósticas gerais de câncer, destacaram-se: mama $(30 \%)$, onco-hematológico $(22 \%)$ e gastrointestinal $(17,5 \%)$, sendo $59,5 \%$ diagnosticados com doença avançada (estadiamento III e IV).

$\mathrm{Na}$ Tabela 2, encontram-se resultados relativos à incidência de ansiedade e depressão. Com base nos critérios da HADS, é possível constatar que 37,5\% dos pacientes apresentaram quadro de ansiedade e $17 \%$ de depressão. Estes resultados foram maiores do que aqueles mensurados pela GAD-7 ( $n=22 \%$ para transtorno de ansiedade) e próximos aos verificados pelo PHQ-9 ( $n=15 \%$ para sinais e sintomas de depressão maior).

A Tabela 3 reporta o coeficiente de fidedignidade Lambda2, a correlação média entre os itens, o erro padrão da média (EPM), a carga fatorial e a correlação item-total. Nota-se que a escala HADS-D obteve as melhores características psicométricas: (1) coeficiente de fidedignidade $-0,84$; (2) correlação média entre os itens - 0,42; (3) EPM - 1,44; (4) carga fatorial média - 0,65; e (5) correlação item-total média - 0,58. A escala GAD-7 apresentou o segundo melhor resultado: (1) coeficiente de fidedignidade - 0,80; (2) correlação média entre os itens - 0,35; (3) EPM - 1,61; (4) carga fatorial média - 0,59; e (5) correlação item-total média $-0,52$. Na sequência, a HADS-A indicou: 1) coeficiente de fidedignidade $-0,74$; (2) correlação média entre os itens - 0,28; (3) EPM - 1,63; (4) carga fatorial média 0,53; e (5) correlação item-total média - 0,45. O PHQ-9 obteve a menor qualidade psicométrica: 1) coeficiente de fidedignidade - 0,79; (2) correlação média entre os itens - 0,26; (3) EPM - 1,70; (4) carga fatorial média 0,51; e (5) correlação item-total média - 0,45.

A grande maioria dos itens obteve correlação item-total maior que 0,30 , com exceção dos itens $6 \mathrm{e}$ 9 do PHQ-9, que abordam questões relacionadas a 
sentimento de fracasso e/ou decepção e pensamentos suicidas. Vale destacar que a média obtida para correlação item-total foi superior a 0,40 em todas as escalas, sendo que HADS-D e a GAD-7 obtiveram melhores valores (acima de 0,50).

Os resultados da análise fatorial mostraram que a maioria dos itens tem uma carga fatorial alta. Para essa análise, utilizou-se como valor de referência 0,30. Esse resultado indicou que os instrumentos avaliados apresentaram evidências de validade ratificadas nas outras análises aqui relatadas. Ao se compararem os resultados obtidos, depreende-se que a HADS-D e a GAD-7 revelaram as melhores características psicométricas. Já o PHQ-9 mostrou a menor qualidade psicométrica, sendo que dois itens obtiveram uma correlação item-total corrigida menor do que 0,30 .

$\mathrm{Na}$ Figura 1, o scree-plot indicou a presença de dois fatores, ao se realizar a análise fatorial com todos instrumentos. Essa análise de eixos principais extraiu dois fatores que são medidos distintamente pelos quatro instrumentos, correspondentes à ansiedade e à depressão no presente estudo.

Para obter evidências de validade discriminante dos instrumentos, foi feita conjuntamente a análise fatorial (Tabela 4). Os resultados apontaram que todos os itens do fator 1 relacionaram-se com o construto de depressão e os itens do fator 2 com o construto de ansiedade. É necessário esclarecer que quatro itens não foram incluídos na Tabela 4 , devido a uma carga

Tabela 1. Caracterização da amostra

\begin{tabular}{|c|c|c|c|c|c|}
\hline \multicolumn{6}{|c|}{ Variáveis demográficas } \\
\hline Sexo & $\mathrm{n}(\%)$ & Estado civil & n ( $\%)$ & Escolaridade & n ( $\%)$ \\
\hline Masculino & $61(30,5)$ & Solteiro & $28(14,0)$ & Fundamental & $29(14,5)$ \\
\hline \multirow{3}{*}{ Feminino } & $139(69,5)$ & Casado & $126(63,0)$ & Médio & $43(21,5)$ \\
\hline & & Divorciado & $24(12,0)$ & Superior & $110(55,0)$ \\
\hline & & Viúvo & $22(11,0)$ & Pós-Graduação & $18(9,0)$ \\
\hline \multicolumn{6}{|c|}{ Variáveis clínicas } \\
\hline Tipo de câncer & & $\mathrm{n}(\%)$ & Estadia & & $\mathrm{n}(\%)$ \\
\hline Mama & & $60(30,0)$ & $\mathrm{I}$ & & $20(10,0)$ \\
\hline Hematológico & & $44(22,0)$ & II & & $45(22,5)$ \\
\hline Gastrointestinal & & $35(17,5)$ & III & & $53(26,5)$ \\
\hline Ginecológico & & $20(10,0)$ & IV & & $66(33,0)$ \\
\hline Pulmão & & $20(10,0)$ & Dados at & & $16(8,0)$ \\
\hline Geniturinário & & $10(5,0)$ & & & \\
\hline Cabeça e pescoço & & $2(1,0)$ & & & \\
\hline Outros & & $9(4,5)$ & & & \\
\hline
\end{tabular}

Tabela 2. Distribuição de pacientes por severidade baixa, moderada a severa para ansiedade e depressão

\begin{tabular}{lclc}
\hline \multicolumn{2}{c}{ Escalas de Ansiedade } & \multicolumn{3}{c}{ Escalas de Depressão } \\
\hline \multicolumn{1}{c}{ HADS-A } & \multicolumn{1}{c}{ HADS-D } \\
\hline Baixa (0-7) & $125(62,5 \%)$ & Baixa (0-8) & $166(83,0 \%)$ \\
Moderada a severa (8-14) & $75(37,5 \%)$ & Moderada a severa $(9-14)$ & $34(17,0 \%)$ \\
Média (DP) & $6,7(3,2)$ & Média (DP) & PHQ-9 \\
\hline \multicolumn{1}{c}{ GAD-7 } & & & B \\
\hline Baixa (0-9) & $156(78,0 \%)$ & Baixa (0-9) & $170(85,0 \%)$ \\
Moderada a severa (10-21) & $44(22,0 \%)$ & Moderada a severa $(10-27)$ & $30(15,0 \%)$ \\
Média (DP) & $6,7(3,0)$ & Média (DP) & $5,5(3,7)$ \\
\hline
\end{tabular}


Tabela 3. Coeficiente de fidedignidade, correlação média entre os itens, carga fatorial e correlação item-total corrigido

\begin{tabular}{ccc}
\hline Descrição do item & Carga & Correlação \\
& Fatorial & item-total \\
\hline
\end{tabular}

HADS-A (Lambda2=0,74; correlação média entre os itens=0,28; EPM=1,63)

1. Eu me sinto tenso ou contraído

3. Eu sinto uma espécie de medo, como se alguma coisa ruim fosse acontecer

5. Estou com a cabeça cheia de preocupações

7. Consigo ficar sentado à vontade e me sentir relaxado

9. Eu tenho uma sensação ruim de medo, como um frio na barriga

11. Eu me sinto inquieto, como se não pudesse ficar parado em lugar nenhum

13. De repente, tenho a sensação de entrar em pânico

Média

$\begin{array}{ll}0,56 & 0,48 \\ 0,62 & 0,51 \\ 0,73 & 0,61 \\ 0,57 & 0,47 \\ 0,45 & 0,38 \\ 0,46 & 0,39 \\ 0,35 & 0,30 \\ 0,53 & 0,45\end{array}$

HADS-D (Lambda2=0,84; correlação média entre os itens=0,42; EPM=1,44)

2. Eu ainda sinto gosto pelas mesmas coisas de antes

4. Dou risada e me divirto quando vejo coisas engraçadas

6. Eu me sinto alegre

8. Eu estou lento para pensar e fazer as coisas

10. Eu perdi o interesse em cuidar da minha aparência

12. Fico esperando animado as coisas boas que estão por vir

14. Consigo sentir prazer quando assisto um bom programa de televisão/rádio

Média

$\begin{array}{ll}0,75 & 0,67 \\ 0,75 & 0,66 \\ 0,66 & 0,59 \\ 0,46 & 0,42 \\ 0,58 & 0,53 \\ 0,67 & 0,60 \\ 0,66 & 0,59 \\ 0,65 & 0,58\end{array}$

GAD-7 (Lambda2=0,80; correlação média entre os itens=0,35; EPM=1,61)

1. Sentir-se nervoso/a, ansioso/a ou muito tenso/a

2. Não ser capaz de impedir ou de controlar as preocupações

3. Preocupar-se muito com diversas coisas

4. Dificuldade para relaxar

5. Ficar tão agitado/a que se torna difícil permanecer sentado/a

6. Ficar facilmente aborrecido/a ou irritado/a

7. Sentir medo como se algo horrível fosse acontecer

Média

$\begin{array}{ll}0,69 & 0,60 \\ 0,66 & 0,57 \\ 0,59 & 0,51 \\ 0,63 & 0,56 \\ 0,54 & 0,50 \\ 0,34 & 0,31 \\ 0,70 & 0,59 \\ 0,59 & 0,52\end{array}$

PHQ-9 (Lambda2=0,79; correlação média entre os itens=0,26; EPM=1,70)

1. Pouco interesse ou pouco prazer em fazer as coisas

2. Se sentir 'pra baixo', deprimido/a ou sem perspectiva

3. Dificuldade para pegar no sono ou permanecer dormindo

4. Se sentir cansado/a ou com pouca energia

5. Falta de apetite ou comendo demais

6. Se sentir mal consigo mesmo/a - ou achar que você é um fracasso

7. Dificuldade para se concentrar nas coisas, como ler o jornal ou ver televisão

8. Lentidão para se movimentar ou falar; ou o oposto

9. Pensar em se ferir de alguma maneira ou que seria melhor estar morto/a

Média

$\begin{array}{ll}0,73 & 0,63 \\ 0,72 & 0,61 \\ 0,56 & 0,49 \\ 0,58 & 0,53 \\ 0,35 & 0,31 \\ 0,32 & 0,29 \\ 0,48 & 0,42 \\ 0,59 & 0,51 \\ 0,30 & 0,26 \\ 0,51 & 0,45\end{array}$




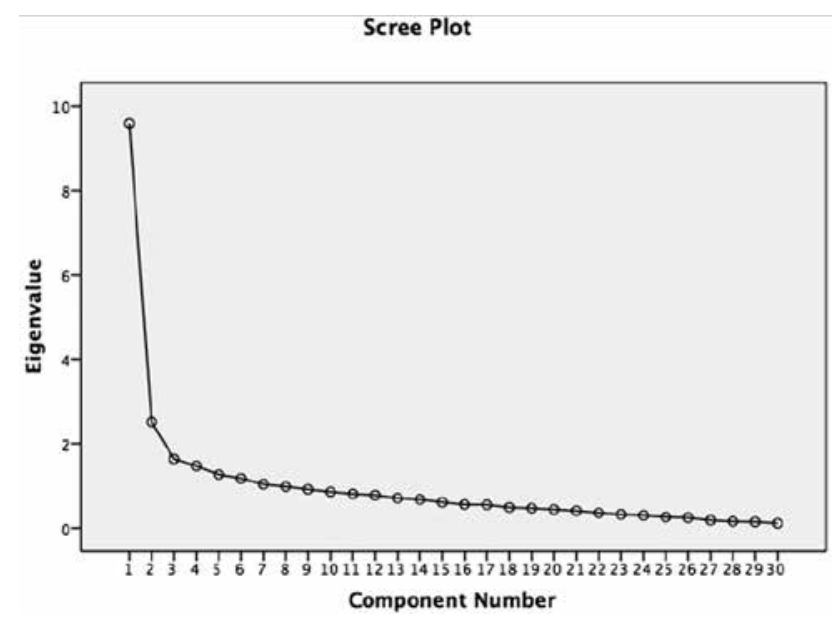

Figura 1. Curva de sedimentação com os eigenvalues obtidos fatorial menor do que 0,32 , sendo que três itens compõem o PHQ-9 e um item compõe a GAD-7. São eles: (PHQ-9) "Dificuldade para pegar no sono ou permanecer dormindo, ou dormir mais do que de costume", "Se sentir mal consigo mesmo/a - ou achar que você é um fracasso ou que decepcionou sua família ou você mesmo/a" e "Pensar em se ferir de alguma maneira ou que seria melhor estar morto/a"; (GAD-7) "Preocupar-se muito com diversas coisas".

A carga fatorial média do Fator 1 foi de 0,61 e do fator 2 , de 0,58 . O coeficiente de fidedignidade Lambda2 do fator 1 foi de 0,90 e do fator 2, de 0,88. Estes valores de fidedignidade foram bem mais elevados do que os alcançados pelos instrumentos separadamente. Os valores da consistência interna sugeriram que em situações

Tabela 4. Análise fatorial dos instrumentos: cargas fatoriais após rotação Promax e extração PAF

\begin{tabular}{lcc}
\hline Itens Escalas & Fator $\mathbf{1}$ & Fator $\mathbf{2}$ \\
\hline 1. PHQ-9. Pouco interesse ou pouco prazer em fazer as coisas & $\mathbf{0 , 7 6}$ & 0,01 \\
2. HADS-D. Eu ainda sinto gosto pelas mesmas coisas de antes & $\mathbf{0 , 7 3}$ & 0,07 \\
12. HADS-D. Fico esperando animado as coisas boas que estão por vir & $\mathbf{0 , 7 1}$ & $-0,11$ \\
4. HADS-D. Dou risada e me divirto quando vejo coisas engraçadas & $\mathbf{0 , 6 9}$ & 0,05 \\
14. HADS-D. Consigo sentir prazer quando assisto um bom programa de televisão/rádio & $\mathbf{0 , 6 9}$ & $-0,06$ \\
10. HADS-D. Eu perdi o interesse em cuidar da minha aparência & $\mathbf{0 , 6 7}$ & $-0,11$ \\
6. HADS-D. Eu me sinto alegre & $\mathbf{0 , 6 2}$ & 0,03 \\
8. HADS-D. Eu estou lento para pensar e fazer as coisas & $\mathbf{0 , 6 0}$ & $-0,14$ \\
8. PHQ-9. Lentidão para se movimentar ou falar, ou o oposto - estar agitado & $\mathbf{0 , 5 9}$ & $-0,03$ \\
4. PHQ-9. Se sentir cansado/a ou com pouca energia & $-0,05$ \\
2. PHQ-9. Se sentir 'pra baixo', deprimido/a ou sem perspectiva & $\mathbf{0 , 5 8}$ & 0,34 \\
5. PHQ-9. Falta de apetite ou comendo demais & $\mathbf{0 , 5 0}$ & 0,34 \\
7. PHQ-9. Dificuldade para se concentrar nas coisas, como ler o jornal ou ver televisão & $\mathbf{0 , 4 8}$ & $-0,19$ \\
Média carga fatorial (n itens = 13) & $\mathbf{0 , 3 8}$ & 0,17 \\
\hline 5. GAD-7. Ficar tão agitado/a que se torna difícil permanecer sentado/a & $\mathbf{0 , 6 1}$ & $\mathbf{0 , 0 0}$ \\
1. GAD-7. Sentir-se nervoso/a, ansioso/a ou muito tenso/a & $-0,18$ & $\mathbf{0 , 7 4}$ \\
7. GAD-7 Sentir medo como se algo horrível fosse acontecer & $-0,05$ & $\mathbf{0 , 7 2}$ \\
11. HADS-A. Eu me sinto inquieto, como se não pudesse ficar parado em lugar nenhum & 0,03 & $\mathbf{0 , 6 9}$ \\
7. HADS-A. Consigo ficar sentado à vontade e me sentir relaxado & $-0,17$ & $\mathbf{0 , 6 5}$ \\
3. HADS-A. Eu sinto uma espécie de medo, como se alguma coisa ruim fosse acontecer & 0,08 & $\mathbf{0 , 6 3}$ \\
4. GAD-7. Dificuldade para relaxar & 0,05 & $\mathbf{0 , 6 2}$ \\
5. HADS-A. Estou com a cabeça cheia de preocupações & 0,08 & $\mathbf{0 , 6 2}$ \\
9. HADS-A. Eu tenho uma sensação ruim de medo, como um frio na barriga & 0,15 & $\mathbf{0 , 6 0}$ \\
2. GAD-7. Não ser capaz de impedir ou de controlar as preocupações & $-0,29$ & $\mathbf{0 , 5 8}$ \\
1. HADS-A. Eu me sinto tenso ou contraído & 0,11 & $\mathbf{0 , 5 7}$ \\
6. GAD-7. Ficar facilmente aborrecido/a ou irritado/a & $-0,03$ & $\mathbf{0 , 5 2}$ \\
13. HADS-A. De repente, tenho a sensação de entrar em pânico & $-0,01$ & $\mathbf{0 , 3 6}$ \\
\hline Média carga fatorial (n itens = 13) & $-0,01$ & $\mathbf{0 , 3 4}$ \\
\hline
\end{tabular}

Nota: Com a extração de dois fatores, $42,7 \%$ da variância total foi explicada; itens com cargas fatoriais $\leq 0,32$ não aparecem na tabela. 
clínicas ou de pesquisas, em que a fidedignidade precisa ser muito alta, é indicada a aplicação de mais de uma medida de depressão ou ansiedade. Os resultados da análise fatorial forneceram evidências positivas da validade discriminante dos construtos como estão sendo medidos pelos instrumentos avaliados. Uma ressalva precisa ser feita em relação ao PHQ-9, em que três itens apresentaram uma relação fraca com o construto de depressão.

Por fim, a correlação entre os instrumentos foi calculada (ver Tabela 5). Os intervalos de confiança de $95 \%$ indicaram que a correlação obtida entre os instrumentos que avaliaram o mesmo construto foi significativamente mais alta do que a correlação entre aqueles que mensuraram outro construto. A correlação entre a HADS-A e a GAD-7 (medidas de ansiedade) foi um pouco mais elevada $(r=0,87)$ do que a correlação $(r=0,82)$ da HADS-D e do PHQ-9 (medidas de depressão). Estes resultados reafirmam a validade discriminante dos instrumentos.

\section{Discussão}

As escalas apresentaram índices de consistência interna recomendáveis para instrumento de triagem, com alto coeficiente de fidedignidade e evidência de validade discriminante, na amostra estudada. Quanto a esse aspecto, a análise fatorial indicou validade de construto composta por dois fatores relacionados a depressão e ansiedade para todas as escalas examinadas. Esse mesmo resultado foi identificado no scree-plot. $\mathrm{O}$ resultado derivado da HADS converge àquele identificado por Moorey e cols. (1991) e contesta outros estudos (Brandberg e cols., 1992; Razavi e cols., 1990; Rodgers e cols., 2005), denotando que se trata de uma escala bidimensional.

Em outra análise, verificou-se que a correlação obtida entre as escalas que avaliaram o mesmo construto foi mais alta, quando comparada com a análise entre escalas que avaliaram diferentes construtos, evidenciando validade discriminante. Isso pode ser explicado pelo fato de que ambos os construtos são medidos de maneira diferente pelas escalas (fator 1 versus fator 2). O PHQ-9 e a GAD-7 norteiam-se pelos critérios do DSM-IV e medem um espectro heterogêneo de sintomas de depressão maior e de transtorno de ansiedade, em contraste com a escala HADS que enfatiza aspectos emocionais da depressão e da ansiedade.

Quando os escores de gravidade clínica preconizados pela literatura foram empregados, a subescala HADS-A identificou $15,5 \%$ a mais de pacientes com indicadores emocionais de ansiedade do que a escala GAD-7. Uma diferença menor $(2 \%)$ foi notada quando se compararam as notas de corte recomendadas para depressão (HADS-D e PHQ-9). A pontuação média foi igual para HADS-A e GAD-7 e ligeiramente diferente para HADS-D e PHQ-9. Além disso, essa incidência foi compatível com aquela encontrada na literatura (Ballenger e cols., 2001).

A presença de ansiedade e depressão entre os pacientes oncológicos, bem como suas implicações negativas na vivência do câncer, ratifica a importância de se identificar instrumentos adequados para avaliação e diagnóstico. A literatura recomenda que sejam breves, ofereçam informações relevantes e sejam capazes de auxiliar os profissionais de saúde na elaboração de um diagnóstico diferencial entre um quadro normal e patológico (Mitchell, 2010). Entre as características psicométricas averiguadas, observou-se que HADS-D e GAD-7 apresentaram os melhores resultados. Entretanto, HADS-A e PHQ-9 também se mostraram adequados para avaliação da ansiedade e da depressão. Cabe lembrar que Hall e cols. (1999) e Mystakidou e cols. (2007) constataram melhor performance da HADS-A, diferentemente do que se evidenciou na presente pesquisa. Essa discrepância pode ter sido acarretada por diversidade de natureza cultural ou clínica, considerando que o estudo de Mystakidou e cols. (2007) foi realizado na Grécia e o estudo de Hall e cols. (1999) incluiu apenas pacientes com câncer de mama.

Tabela 5. Correlações entre os instrumentos com intervalos de confiança de $95 \%$

\begin{tabular}{lcc}
\hline Escalas & Correlação & Intervalo de confiança de 95\% \\
\hline HADS-A - GAD-7 & 0,87 & $0,83-0,90$ \\
HADS-D - PHQ-9 & 0,82 & $0,75-0,87$ \\
HADS-A - PHQ-9 & 0,58 & $0,49-0,67$ \\
HADS-D - GAD-7 & 0,55 & $0,44-0,64$ \\
HADS-A - HADS-D & 0,50 & $0,38-0,61$ \\
\hline
\end{tabular}


Apenas quatro itens tiveram carga fatorial e correlação item-total abaixo daquela apontada pela literatura (Clark \& Watson, 1995; Costello \& Osborne, 2005; Laros, 2012; Reise, Waller \& Comrey, 2000). Atribuem-se a esse resultado, as diferenças pontuadas pela literatura quanto a sintomas de depressão e sintomas decorrentes da doença e do tratamento, bem como diferenças entre sintomas de depressão primária e de depressão secundária. Em estudo realizado por Li, Fitzgerald e Rodin (2012), foi possível verificar que a depressão secundária ao câncer está menos associada a pensamentos depressivos, como culpa e fracasso, quando comparada a depressão primária $(\mathrm{Li}$ e cols., 2012).

Ao contrário do que enfatizaram Cosco e cols. (2012), mas em consonância com o trabalho conduzido por Moorey e cols. (1991), constatou-se que a HADS pode ser uma ferramenta adequada e útil para ser empregada em serviços especializados de oncologia. É importante realçar que uma avaliação sistemática e bem fundamentada de pacientes oncológicos fornece indicadores para manejo dos aspectos psicossociais envolvidos na vivência do câncer. Tal monitoramento permite a identificação precoce de transtornos, instrumentalizando a equipe de saúde - e especialmente o psicólogo - no reconhecimento de prioridades de uma conduta terapêutica centrada nas necessidades e demandas do paciente. Responder às urgências e optar corretamente em casos de encaminhamento, é crucial na medida em que se defendem cuidados preventivos e proativos, mesmo em nível de assistência de alta complexidade. Sendo assim, é preciso conjugar eficiência e eficácia terapêutica atendendo bem um número crescente de pacientes. Uma rigorosa triagem torna-se essencial neste cenário.

Esta investigação comportou algumas limitações como a composição e o tamanho da amostra. De fato, os participantes foram selecionados em um centro especializado de uma única localidade brasileira, o que pode ocasionar a reprodução de características socioculturais específicas, reduzindo o grau de generalização dos resultados aqui divulgados.

Recomendam-se, então, estudos multicêntricos em diferentes centros e regiões do país. Tais iniciativas igualmente propiciariam comparar um número maior e mais diversificado de cânceres em estadiamentos diferentes. No âmbito internacional, sabe-se que câncer de pâncreas, pulmão e orofaringe predispõem à depressão (Li e cols., 2012). O estadiamento clínico da doença também pode constituir um fator preditivo para transtorno de ansiedade e de depressão, assim como, idade, estado civil e gênero do paciente (Li e cols., 2012).

A presente investigação verificou que os instrumentos HADS, GAD-7 e PHQ-9 apresentaram boas características psicométricas. Estes resultados sugerem adequação dos instrumentos para avaliação diagnóstica de ansiedade e depressão em pacientes oncológicos, desde a triagem em um centro especializado em oncologia. Com esta pesquisa reafirma-se a necessidade de futuros estudos que enfoquem as evidências de validade da HADS, GAD-7 e PHQ-9 para pacientes oncológicos no Brasil, bem como trabalhos que contemplem a sensibilidade e a especificidade das escalas. Traçar um panorama nacional da população acometida por um ou mais cânceres subsidiará programas mais eficazes de acompanhamento clínico neste campo da saúde.

\section{Referências}

Adler, N. E., \& Page, A. E. K. (Eds.). (2008). Cancer care for the whole patient: meeting psychosocial health needs. Washington, DC: Institute of Medicine of the National Academies Press.

Baker-Glenn, E., Mitchell, A. J., \& Symonds, P. (2008). Screening using the emotion thermometers: a useful extension to the distress thermometer when identifying major and minor depression? Psycho-Oncology, 17(suppl. 2), S57. DOI: 10.1002/pon.1389.

Ballenger, J. C., Davidson, J. R. T., Lecrubier, Y., Nutt, D. J., Jones, R. D., \& Berard, R. M. F. (2001). Consensus statement on depression, anxiety, and Oncology. Journal of Clinical Psychiatry, 62(suppl. 8), 64-67.

Botega, N. J., Bio, M. R., Zomignani, M. A., Garcia Jr, C., \& Pereira, W. A. B. (1995). Transtorno de humor em enfermaria de clínica médica e validação de escala de medida (HAD) de ansiedade e depressão. Revista de Saúde Pública, 29(5), 355-363. DOI: 10.1590/S0034-89101995000500004.

Brandberg, Y., Bolund, C., Sigurdardottir, V., Sjoden, P. O., \& Sullivan, M. (1992). Anxiety and depressive symptoms at different stages of malignant melanoma. Psycho-Oncology, 1, 71-78. DOI: 10.1002/ pon.2960010204.

Bultz, B. D., \& Johansen, C. (2011). Screening for distress, the 6th vital sign: where are we, and where are we going? Psycho-Oncology, 20(6), 569-571. DOI: 10.1002/pon.1986. 
Cattell, R. B. (1966). The scree test for the number of factors. Multivariate Behavioral Research, 1, 245-276. DOI: $10.1207 / \mathrm{s} 15327906 \mathrm{mbr} 0102 \_10$.

Clark, A. C., \& Watson, D. (1995). Constructing validity: basic issues in objective scale development. Psychological Assessment, 7, 309-319. DOI: 10.1037/ 1040-3590.7.3.309.

Cosco, T. D., Doyle, F., Ward, M., \& McGee, H. (2012). Latent structure of the Hospital Anxiety and Depression Scale: a 10-year systematic review. Journal of Psychosomatic Research, 72, 180-184. DOI: 10.1016/j.jpsychores.2011.06.008.

Costello, A. B., \& Osborne, J. W. (2005). Best practices in exploratory factor analysis: Four recommendations for getting the most from your analysis. Practical Assessment, Research \& Evaluation, 10, 1-9. DOI: 10.1.1.110.9154.

Coyne, J. C., \& Sonderen, E. V. (2012a). No further research needed: abandoning the Hospital Anxiety and Depression Scale (HADS). Journal of Psychosomatic Research, 72, 173-174. DOI: 10.1016/j. jpsychores. 2011.12.003.

Coyne, J. C., \& Sonderen, E. V. (2012b). The Hospital Anxiety and Depression Scale (HADS) is dead, but like Elvis, there will still be citing. Journal of Psychosomatic Research, 73, 77-78. DOI: 10.1016/j. psychores.2012,04.002.

Dalton, S. O., Laursen, T. M., Ross, L., Mortensen, P. B., \& Johansen, C. (2009). Risk for hospitalization with depression after cancer diagnosis: a nationwide, population-based study of cancer patients in Denmark from 1973-2003. Journal of Clinical Oncology, 27, 1440-1445. DOI: 10.1200/JCO.2008.20.5526.

Damásio, B. F. (2012). Uso da análise fatorial exploratória em Psicologia. Avaliaşão Psicológica, 11, 213-228.

Decat, C. S., Laros, J. A., \& Araujo, T. C. C. F. (2009). Termômetro de distress: validação de um instrumento breve para avaliação diagnóstica de pacientes oncológicos. Psico-USF, 14(3), 253-260. DOI: $10.1590 /$ S1413-82712009000300002

Grady-Weliky, T. A. (2002). Comorbidity and its implications on treatment: change over the life span. Syllabus and Proceedings Summary, American Psychiatric Association, Annual Meeting, 295.

Hall, A., A’Hern, R., \& Fallowfield, L. (1999). Are we using appropriate self-report questionnaires for detecting anxiety and depression in women with early breast cancer? European Journal of Cancer, 35, 79-85.

Jacobsen, P. B., Holland, J. C., \& Steensma, D. P. (2012). Caring for the whole patient: the science of psychosocial care. Journal of Clinical Oncology, 30(12), 1-3. DOI: 10.1200/JCO.2011.41.4078.

Kroenke, K., Spitzer, R. L., \& Williams, J. B. W. (2001). The PHQ-9: validity of a brief depression severity measure. Journal of General Internal Medicine, 16, 606613. DOI: $10.1046 / j .1525-1497.2001 .016009606 . x$

Kroenke, K., Spitzer, R. L., Williams, J. B. W., Monahan, P. O., \& Löwe, B. (2007). Anxiety disorders in primary care: prevalence, impairment, comorbidity, and detection. Annals of Internal Medicine, 146, 317-325.

Kroenke, K., Spitzer, R. L., Williams, J. B. W., \& Lowe, B. (2010). The patient health questionnaire somatic, anxiety, and depressive symptom scales: a systematic review. General Hospital Psychiatry, 32, 345-359.

Lambert, S. D., Pallant, J. F., Boyes, A. W., King, M. T., Britton, B., \& Girgis, A. (2013). A rasch analysis of the hospital anxiety and depression scale (HADS) among cancer survivors. Psychological Assessment, 25(2), 379-390. DOI: 10.1037/a0031154.

Laros, J. A. (2012). O uso da análise fatorial: algumas diretrizes para pesquisadores. Em L. Pasquali (Org.), Análise fatorial para pesquisadores (pp. 141-160). Brasília: LabPAM Saber e Tecnologia.

Levin, T. T., \& Alici, Y. (2010). Anxiety disorders. Em J. C. Holland, W. S. Breitbart, P. B. Jacobsen, M. S. Lederberg, M. J. Loscalzo, \& R. McCorkle (Eds.), Psycho-Oncology (2a ed.) (pp. 324-331). Nova Iorque, NI: Oxford University Press.

Li, M., Fitzgerald, P., \& Rodin, G. (2012). Evidencebased treatment of depression in patients with cancer. Journal of Clinical Oncology, 30(11), 11871196. DOI: 10,1200/JCO.2011.39.7372.

Mapi Research Institute. (2006). Certificate of linguistic validation certificate: general anxiety disorder-7 (GAD-7). Lyon, FR: Mapi Research Institute.

Massie, M. J. (1989). Anxiety, panic, and phobias. Em J. C. Holland, \& J. H. Rowland (Org.), Handbook of Psychooncology: psychological care of the patient with cancer 
(pp. 300-309). Nova Iorque, NI: Oxford University Press.

Massie, M. J. (2004). Prevalence of depression in patients with cancer. Journal of the National Cancer Institute Monographs, 32, 57-71. DOI: 10.1093/ jncimonographs/lgh014.

Miller, K., \& Massie, M. J. (2010). Depressive disorders. Em J. C. Holland, W. S. Breitbart, P. B. Jacobsen, M. S. Lederberg, M. J. Loscalzo, \& R. McCorkle (Eds.), Psycho-Oncology (2a ed.) (pp. 311-318). Nova Iorque, NI: Oxford University Press.

Mitchell, A. J. (2010). Screening procedures for psychosocial distress. Em J. C. Holland, W. S. Breitbart, P. B. Jacobsen, M. S. Lederberg, M. J. Loscalzo, \& R. McCorkle (Eds.), Psycho-Oncology (2a ed.) (pp. 389398). Nova Iorque, NI: Oxford University Press.

Moorey, S., Greer, S., Watson, M., Gorman, C., Rowden, L., Tunmore, R., ... \& Bliss, J. (1991). The factor structure and factor stability of the Hospital Anxiety and Depression Scale in patients with cancer. British Journal of Psychiatry, 158, 255-259.

Mystakidou, K., Tsilika, E., Parpa, E., Galanos, A., \& Vlahos, L. (2007). Psychometric properties of the impact of event scale in greek cancer patients. Journal of Pain and Symptom Management, 33(4), 340-347. DOI: $10.1016 /$ j.painsymman.2006.09.023.

Noyes Jr., R., Holt, C. S., \& Massie, M. J. (1998). Anxiety disorders. Em J. C. Holland (Eds.), Psycho-oncology (pp. 548-563). Nova Iorque, NI: Oxford University Press.

Osório, F. L., Mendes, A. V., Crippa, J. A. S., \& Loureiro, S. R. (2009). Study of the discriminative validity of the PHQ-9 and PHQ-2 in a sample of Brazilian women in the context of primary health care. Perspectives in Psychiatric Care, 45, 216-227.

Pirl, W. F. (2010). Instruments in psycho-oncology. Em J. C. Holland, W. S. Breitbart, P. B. Jacobsen, M. S. Lederberg, M. J. Loscalzo, \& R. McCorkle (Eds.), Psycho-Oncology ( ${ }^{\mathrm{a}}$ ed.) (pp. 119-130). Nova Iorque, NI: Oxford University Press.

Razavi, D., Delvaux, N., Farvacques, C., \& Robaye, E. (1990). Screening for adjustment disorders and major depressive disorder in cancer patients: a clinical model. British Journal of Psychiatry, 156, 79-83.
Reise, S. P., Waller, N. G., \& Comrey, A. L. (2000). Factor analysis and scale revision. Psychological Assessment, 12, 287-297. DOI: 10.1037/1040-3590.12.3.287.

Rodgers, J., Martin, C. R., Morse, R. C., Kendell, K., $\&$ Verril, M. (2005). An investigation into the psychometric properties of the Hospital Anxiety and Depression Scale in patients with breast cancer. Health and Quality of Life Outcomes, 3, 41-53. DOI: 10.1186/1477-7525-3-41.

Smith, G. T., \& McCarthy, D. M. (1995). Methodological considerations in the refinement of clinical assessment instruments. Psychological Assessment, 7, 300-308. DOI: 10.1037/1040-3590.7.3.300.

Spitzer, R. L., Kroenke, K., \& Williams, J. B. W. (1999). Validation and utility of a self-report version of PRIME-MD: the PHQ primary care study. Journal of the American Medical Association, 282(18), 1737-1744.

Spitzer, R. L., Williams, J. B., Kroenke, K., Linzer, M., DeGruy, F. V., Hahn, S. R., ... \& Johnson, J. G. (1994). Utility of a new procedure for diagnosing mental disoreders in primary care. The PRIMEMD 1000 study. Journal of the American Medical Association, 272(22), 1749-1756.

Spitzer, R. L., Kroenke, K., Williams, J. B. W., \& Löwe, B. (2006). A brief measure for assessing generalized anxiety disorder: the GAD-7. Archives of Internal Medicine, 166(10), 1092-1097.

Stark, D., Kiely, M., Smith, A., Velikova, G., House, A., \& Selby, P. (2002). Anxiety disorders in cancer patients: their nature, associations, and relation to quality of life. Journal of Clinical Oncology, 20(14), 3137-3148. DOI: 10.1200/JCO.2002.08.549.

Tabachnick, B. G., \& Fidell, L. S. (2007). Using multivariate statistics ( $5^{\mathrm{a}}$ ed.). Boston: Person Education.

Tellegen, P. J., \& Laros, J. A. (2004). Cultural bias in the SON-R test: comparative study of Brazilian and Dutch children. Psicologia: Teoriae Pesquisa, 20(2), 103111. DOI: $10.1590 /$ S0102-37722004000200002.

Ten Berge, J. M. F., \& Zegers, F. E. (1978). A series of lower bounds to the reliability of a test. Psychometrika, 43(4), 575-579.

Recebido em: 27/04/2013

Reformulado em: 18/09/2013

Segunda reformulação em: 10/12/2013

Aprovado em: 03/02/2014 
Sobre os autores:

Cristiane Decat Bergerot é doutora em Psicologia da Saúde pela Universidade de Brasília (UnB), especialista em Psicologia da Saúde e Hospitalar pela Associação de Combate ao Câncer de Goiás (ACCG), com certificação em Psico-Oncologia pela Sociedade Brasileira de Psico-Oncologia (SBPO); e psicóloga responsável pelo serviço de Psico-Oncologia do Centro de Câncer de Brasília (CETTRO).

Jacob Arie Laros é PhD em Personality and Educational Psychology pela Rijksuniversiteit Groningen (RuG) Holanda. É professor associado IV no Instituto de Psicologia da Universidade de Brasília (UnB) e coordenador do laboratório META (Métodos e Técnicas de Avaliação). Suas áreas de expertise são: análise de dados, testes de habilidades cognitivas, avaliação educacional em larga escala e avaliação de programas educacionais e sociais.

Tereza Cristina Cavalcanti Ferreira de Araujo é doutora pela Université de Paris X-Nanterre e pós-doutora pela Unesco. É pesquisadora do CNPq e coordenadora do Laboratório de Saúde e Desenvolvimento Humano (UnB), no qual realiza pesquisas sobre: processo saúde-doença; relações sociais na assistência à saúde; psicologia aplicada à oncologia e reabilitação; transição, condições especiais e qualidade de vida; saúde e gênero.

Contato com os autores:

Universidade de Brasília - Instituto de Psicologia - Laboratório de Saúde e Desenvolvimento Humano - 70910-900 Brasília, DF.

E-mails: crisdecat@yahoo.com.br; laros@unb.br; araujotc@unb.br 
